\title{
No visibilización del Instituto Nacional de Salud del Perú en los análisis de la producción científica peruana publicados por CONCYTEC
} Non-visibility of the National Institute of Health of Peru in the analyzes of Peruvian
scientific production published by CONCYTEC

\author{
Franco Romaní Romaní $i^{1, a}$ \\ 1 Facultad de Medicina Humana, Universidad de Piura. Lima, Perú \\ ${ }^{\text {a }}$ Médico cirujano, magister en epidemiología. ORCID: https://orcid.org/0000-0002-6471-5684
}

Correspondencia:

Franco Romani Romaní

franco.romani@udep.edu.pe

Recibido: 31 de agosto 2021

Aprobado: 14 de septiembre 2021

Publicación en línea: 25 de septiembre 2021

Conflictos de interés: El autor declara que es Editor Cientifico de la Revista Peruana de Medicina Experimental y Salud Pública del Instituto Nacional de Salud.

Fuente de financiamiento:

Autofinanciado

Citar como: Romani F. No visibilización del Instituto Nacional de Salud del Perú en los análisis de la producción cientifica peruana publicados por CONCYTEC. An Fac med. 2021;82(3):244-6. DOI: https://doi. org/10.15381/anales.v82i3.21138
An Fac med. 2021;82(3):244-6. / DOI: https://doi.org/10.15381/anales.v82i3.21138

Sr. Editor,

En el 2014, el Consejo Nacional de Ciencia, Tecnología e Innovación Tecnológica (CONCYTEC) publicó un análisis que cuantificó las publicaciones de las instituciones de investigación del Perú para el periodo 2006-2011 ${ }^{(1)}$. Este informe fue la primera aproximación oficial de la producción científica de instituciones peruanas; sin embargo, no visibilizó a una institución de más de 100 años de existencia como el Instituto Nacional de Salud (INS) del Perú.

EI CONCYTEC es el ente rector del Sistema Nacional de Ciencia, Tecnología e Innovación (Sinacti), entre sus funciones debe calificar a las entidades del Sinacti; y reconocerles su contribución. Forman parte del Sinacti los Institutos Públicos de Investigación (IPI), entre los cuáles está el INS ${ }^{(2)}$, que además es el ente rector en investigación en salud. Ante la falta de visibilidad del INS en el análisis publicado el $2014^{(1)}$, y en vista de la creciente importancia de las publicaciones científicas para evaluar el desempeño y contribución de las instituciones ${ }^{(3)}$; se evaluó las publicaciones de CONCYTEC que han analizado la producción científica peruana con el fin de determinar si se ha cuantificado el número de publicaciones del INS.

Se consultó el Repositorio Nacional Digital de Ciencia, Tecnología e Innovación (ALICIA) en agosto del 2021, mediante el buscador avanzado (https://alicia.concytec.gob.pe/vufind/ Search/Advanced) con las siguientes estrategias de búsqueda: a. «indicador bibliométrico» y «Perú», b. «producción científica» y «Perú»; y c. «publicaciones» y «Perú». Además, en todas las estrategias se usó el término «Consejo Nacional de Ciencia, Tecnología e Innovación Tecnológica» para el campo de «autor». La búsqueda se hizo para la coincidencia de todos los términos, sin límites por año, lenguaje y formato.

Se encontró dos publicaciones de CONCYTEC que midieron la producción científica de las instituciones peruanas con técnicas bibliométricas (denominados ítem 1 y 2$)^{(1,4)}$. Ambos estudios fueron encargados al SCImago Research Group. Entre ambas publicaciones se analizó un periodo de 12 años y usaron como fuente de información la base de datos Scopus y la herramienta SCImago Journal \& Country Rank. Se corroboró que no se visibilizó al INS, ni se cuantificó su producción científica en el periodo 2006 al 2017 (Tabla 1).

En el ítem 1, publicado el 2019, los autores decidieron incluir la producción científica del INS y otras cuatro instituciones, como producción del Ministerio de Salud (Minsa) ${ }^{(4)}$. En el ítem 2 (publicación del 2014) ${ }^{(1)}$ no se presentó una descripción metodológica y regla de decisión para estas agrupaciones, se asume que los autores optaron por la misma estrategia que el ítem 1. 
Al respecto, el INS tiene un identificador en la base de datos Scopus (Affiliation ID: 60071247), y su denominación normalizada es «Instituto Nacional de Salud, Lima». En la jerarquía de afiliación se encuentra como institución hija del «Ministerio de Salud, Lima» (Affiliation ID: 60071228). Sin embargo, esta situación no explica su ausencia como una firma institucional independiente; puesto que las dos publicaciones analizadas presentan en el sector salud la producción científica de varios perfiles institucionales que según Scopus también son instituciones hijas del Minsa (Hospital Nacional
Cayetano Heredia, Hospital Nacional Dos de Mayo, Hospital Nacional Arzobispo, Loayza, Hospital Nacional Docente Madre Niño San Bartolomé, Instituto Nacional de Enfermedades Neoplásicas).

Según la organización del Minsa, el INS es un organismo público adscrito, y no forma parte de sus órganos de administración interna, órganos de apoyo, de línea, ni órganos desconcentrados; en esta última categoría se encuentran los hospitales e institutos nacionales especializados, los cuáles sí fueron visibilizados con su contribución científica. En el análisis del sector de instituciones del gobierno, tampoco se consideró al INS, a pesar que es un IPI (Tabla 1).

Actualmente, las instituciones de investigación se encuentran muy interesadas en el número de sus publicaciones. Dicho atributo es un factor para acceder a fondos de investigación, cumplir con sistemas de acreditación, para fortalecer el liderazgo en una determinada disciplina, o cimentar el prestigio institucional ${ }^{(3)}$. Así, la adecuada representación de la filiación institucional se ha convertido en parte de la formación en integridad científica ${ }^{(5)}$; sin embargo, en

Tabla 1. Análisis comparativo de las publicaciones de CONCYTEC que midieron la producción científica en el Perú respecto a la identificación de las publicaciones del Instituto Nacional de Salud.

\begin{tabular}{ll} 
Característica & \multicolumn{1}{c}{ Ítem 1} \\
\hline Fecha de publicación & Diciembre 2019 \\
\hline Equipo de investigación & SCImago Research Group \\
\hline ISBN & $978-9972-50-182-1$ \\
\hline Periodo de análisis & 2012 a 2017 \\
\hline & Presenta la sección metodología (pág. 14) que describe \\
& la normalización de la información. Se describe los \\
& sectores institucionales en base al Manual de Frascati. \\
& Las categorías de sectores fueron: universidades, salud \\
& (que incluye hospitales y clínicas al país, sin importar \\
naturaleza pública, privada o universitaria), gobierno \\
(compuesto por organismos del gobierno central y de \\
gobiernos regionales; por ejemplo, los ministerios, así \\
como los institutos públicos de investigación, entre \\
otros), privado yotros (comprende instituciones sin fines \\
de lucro, organismos internacionales).
\end{tabular}

Setiembre 2014

SClmago Research Group

978-9972-50-192-0

2006 a 2011

Las notas metodológicas (pág. 89) no describen los criterios utilizados para agrupar a las instituciones de investigación. Se describe que la fuente de información es Scopus y la herramienta de acceso abierto SCImago Journal \& Country Rank. La metodología se centra en presentar los indicadores bibliométricos en tres bloques: la dimensión cuantitativa, la dimensión cualitativa, y la dimensión estructural y de relaciones de la producción científica.

En la sección 5 de presentación de la producción científica por instituciones, se presentan los siguientes sectores: empresas, universidades, instituciones biomédicas, del gobierno y otras.

Fueron identificadas 62 instituciones en este sector que han publicado al menos un artículo en revistas Scopus. Se identifica al Minsa ( $n=825)$, como la única institución con más de 500 documentos publicados, seguido del Hospital Nacional Cayetano Heredia $(n=254)$ y el Naval

Resultados para las instituciones del sector salud

Resultados para las instituciones del sector gobierno
Medical Research Center Detachment $(n=223)$. Los autores asignan al Minsa la producción científica del Instituto Nacional de Salud, el Instituto Nacional de Salud del Niño, el Instituto Nacional de Ciencias Neurológicas, el Instituto Nacional Materno Perinatal y el Instituto Nacional de Salud Mental Honorio Delgado-Hideyo Noguchi.

Fueron identificadas 29 instituciones gubernamentales con al menos un artículo publicado en revistas Scopus. Ninguna supera 500 publicaciones en el periodo analizado. Entre las 16 instituciones identificadas (tabla 62) con más de 10 publicaciones en el periodo, siete son Institutos Públicos de Investigación, sin embargo, no se identifica al Instituto Nacional de Salud. Las tres primeras fueron el Instituto del Mar del Perú ( $n=190)$, el Instituto Geofísico del Perú ( $n=174$ ) y el Instituto de Investigación de la Amazonía Peruana ( $n=104)$.
Se les denominó instituciones biomédicas. La tabla 19 presenta como la de mayor producción al Minsa ( $n=427)$, seguido por Naval Medical Research Center Detachment ( $n=156)$ y el Hospital Nacional Cayetano Heredia $(n=85)$. No se realiza una descripción sobre que instituciones comprendía el Minsa. Tampoco se visibiliza al Instituto Nacional de Salud como institución de investigación.

La tabla 2 presenta 21 instituciones ordenadas según el número de publicaciones. Las tres primeras fueron el Instituto del Mar del Perú ( $n=102)$, el Instituto Geofísico del Perú ( $n=88$ ) y el Instituto de Investigación Nutricional $(n=71)$. La tabla identifica a siete Institutos Públicos de Investigación, pero no se visibiliza al Instituto Nacional de Salud. 
este caso la inadecuada firma institucional de los autores no fue el problema. Según Scopus, al 13 de agosto 2020 había 848 documentos correspondientes al INS, esta cantidad es el $20,5 \%$ de las publicaciones atribuidas al Minsa. Así, el INS tiene la mayor cantidad de publicaciones entre las catalogadas como instituciones hijas del Minsa. Esta tendencia fue ratificada en un análisis de las publicaciones originales del INS recuperadas en Scopus y en Scielo Perú para el periodo 1998 al 2018, que encontró 618 artículos y una tendencia creciente de su producción ${ }^{(6)}$.

Se concluye que los dos estudios del ente rector del Sinacti que han cuantificado la producción científica de las instituciones de investigación en el Perú, no han visibilizado la contribución del INS; ni en su rol como institución de investigación del sector salud, ni como un IPI. Todas las instituciones del Sinacti deben ser reconocidas por su desempeño en las actividades de ciencia, tecnología e innovación.

\section{REFERENCIAS BIBLIOGRÁFICAS}

1. Principales indicadores bibliométricos de la actividad científica peruana, 2006-2011. Consejo Nacional de Ciencia, Tecnologia e Innovación Tecnológica - CONCYTEC. [Internet]. Lima, Perú.: CONCYTEC; 2014. Informe N¹. Disponible en: https:// cdn.www.gob.pe/uploads/document/file/747524/ Informe1_Principales_Indicadores_2006_2011.pdf

2. Ley del Sistema Nacional de Ciencia, Tecnología e Innovación (SINACTI). [Internet]. Ley N³1250. jul 2, 2021. Disponible en: https://busquedas.elperuano. $\mathrm{pe} / \mathrm{download} / \mathrm{url} / \mathrm{ley}$-del-sistema-nacional-de-ciencia-tecnologia-e-innovacion-ley-n-31250-1968664-1
3. Thonon F, Boulkedid R, Delory T, Rousseau S, Saghatchian M, van Harten W, et al. Measuring the Outcome of Biomedical Research: A Systematic Literature Review. Fanelli D, editor. PLOS ONE. 2015;10(4):e0122239. DOI: 10.1371/journal. pone.0122239

4. Principales indicadores bibliométricos de la actividad científica peruana, 2012-2017. [Internet]. Lima, Perú.: Consejo Nacional de Ciencia Tecnologia e Innovación Tecnológica (CONCYTEC); 2019. Informe $N^{\circ} 5$. Disponible en: http://portal. concytec.gob.pe/images/publicaciones/informes/ Principales_indicadores_bibliomtricos_de_la_actividad_cientfica_peruana_SCIMAGO.pdf

5. Bachelet VC, Uribe FA, Diaz RA, Vergara AF, BravoCórdova F, Carrasco VA, et al. Author misrepresentation of institutional affiliations: protocol for an exploratory case study. BMJ Open. 2019;9(2):e023983. DOI: 10.1136/bmjopen-2018-023983

6. Romani F. Análisis bibliométrico de las publicaciones científicas originales del Instituto Nacional de Salud del Perú en el periodo 1998-2018. Rev Peru Med Exp Salud Pública. 2020;37(3):485-94. DOI: 10.17843/rpmesp.2020.373.5470 